\title{
Differential Signal Transduction of Alternatively Spliced FGFR2 Variants Expressed in Human Mammary Epithelial Cells
}

\author{
ALLISON B. MOFFA ${ }^{1}$ AND STEPHEN P. ETHIER ${ }^{2,3 *}$ \\ ${ }^{1}$ Cellular and Molecular Biology Graduate Program, University of Michigan, \\ Ann Arbor, Michigan \\ ${ }^{2}$ Breast Cancer Program, Barbara Ann Karmanos Cancer Institute, \\ Detroit, Michigan \\ ${ }^{3}$ Department of Pathology, Wayne State University School of Medicine, \\ Detroit, Michigan
}

\begin{abstract}
Gene amplification and protein overexpression of fibroblast growth factor receptor 2 (FGFR2) characterize the SUM-52 breast cancer cell line developed in our laboratory. SUM-52 cells express nine distinct alternatively spliced isoforms of FGFR2. Among these isoforms are two otherwise identical FGFR2 variants that express either the $\mathrm{C} 1$ or $\mathrm{C} 3$ carboxyl terminus. FGFR2-C3 variants are not normally expressed by human mammary epithelial (HME) cells, and we have shown that overexpression of FGFR2-C3 in HME cells results in potent transformation. In particular, FGFR2-C3 expression leads to robust levels of constitutively tyrosine phosphorylated FRS2 in the absence of ligand stimulation. In contrast, overexpressed FGFR2-C1 requires constant stimulation with exogenous keratinocyte growth factor (KGF) to mimic the signaling capability of FGFR2-C3. However, activation of FRS2 that results from KGF-stimulated FGFR2-C1 signaling is transient and is associated with a mobility shift of FRS2 not observed when this signaling molecule is activated by the C3 isoform of FGFR2. Mutation of the only tyrosine phosphorylated site present in the C1 terminus and absent from C3, Tyr769, did not yield a receptor that rivaled the potent signaling of FGFR2-C3. We therefore conclude that aberrant expression of alternatively spliced isoforms of FGFR2 with the C3 carboxyl terminus in the SUM-52 breast cancer cells results in sustained activation of signal transduction leading to transformation. J. Cell. Physiol. 210: 720-731, 2007.
\end{abstract}

(c) 2006 Wiley-Liss, Inc.

Significant progress has been made toward understanding the role of receptor tyrosine kinase signaling in cancer. Receptor tyrosine kinases can acquire transforming function via gene amplification, protein overexpression, and/or mutations, leading to constitutive activation and aberrant downstream signaling. The fibroblast growth factor receptor (FGFR) family has been found to play a role in the development of several types of cancers. FGFR3 is often mutated in bladder cancer, and occasionally in cervical and colorectal cancers as well (Cappellen et al., 1999; Jang and Park, 2001). In gastric cancers, FGFR1, FGFR2, and FGFR4 have been shown to be overexpressed (Shin et al., 2000). In particular, the splice variant FGFR2-IIIb, which will be discussed in this article, is commonly gene amplified and overexpressed in gastric cancers (Hattori et al., 1990; Itoh et al., 1994). In regard to breast cancer, studies have indicated that FGFR1, FGFR2, and FGFR4 genes are occasionally amplified and overexpressed, with the FGFR2-IIIb gene overexpressed in approximately $5 \%$ of cases (Adnane et al., 1990; PenaultLlorca et al., 1995). Beyond this, however, there is limited understanding of a role of FGFR signaling in human breast cancer.

SUM-52PE is a human breast cancer cell line developed in our laboratory (Ethier et al., 1996). These cells have gene amplification, and protein and message overexpression of FGFR2. Specifically, SUM-52 cells overexpress the IIIb splice variant of FGFR2, which is the version expressed by epithelial cells and is the receptor for keratinocyte growth factor (KGF). The SUM-52 cells overexpress nine distinct isoforms of FGFR2. Although all isoforms contain the Ig-IIIb region in their ectodomain, they differ in the number of the other immunoglobulin-like domains, the pre- sence or absence of the acid box, and the carboxyl terminal region. All three carboxyl termini (C1, C2, and C3) are represented among the FGFR2 isoforms expressed by the SUM-52 breast cancer cells (Tannheimer and Ethier, 2000). The C1 and C2 carboxyl termini are alternatively spliced from the same exon, whereas C3 is expressed from a separate exon (Itoh et al., 1994). Normal human mammary epithelial cells express only the full-length C1 isoform of FGFR2, whereas expression of FGFR2 variants with the C3 carboxyl terminus has only been described in cancer cells. Our work has focused on understanding the differences in transforming function between two fulllength FGFR2 isoforms isolated from the SUM-52 cells that differ only in their carboxyl termini (isoforms C1\#38 and C3\#4). The C3 carboxyl terminus is significantly shorter (11 amino acids in length) than the $\mathrm{C} 1$ terminus ( 64 amino acids). There are 10 amino acids common to both receptors' carboxyl terminal sequence, leaving only one amino acid unique to the C3 tail, and 54 on the C1 (Itoh et al., 1994). The FGFR2-C1 carboxyl terminus includes several tyrosine residues. Among these, only the tyrosine at site 769 is known to

Contract grant sponsor: National Cancer Institute; Contract grant number: RO1 CA 70354.

*Correspondence to: Stephen P. Ethier, PhD, Deputy Director, Barbara Ann Karmanos Cancer Institute, 4100 John R. Street, Detroit, MI 48201. E-mail: ethier@karmanos.org

Received 7 July 2006; Accepted 22 August 2006

DOI: $10.1002 /$ jep. 20880 
be phosphorylated (homologous to Tyr766 on FGFR1), and serves as a binding site for phospholipase C- $\gamma$ (PLC $\gamma)$ as well as the Shb adapter protein, involved in MAPK signaling (Mohammadi et al., 1991, 1996; Cross et al., 2002).

Previously, we have stably overexpressed both FGFR2 full-length variants (referred to as FGFR2-C1 and FGFR2-C3) in H16N2 human mammary epithelial (HME) cells. Our results suggested that FGFR2-C3 is more transforming than FGFR2-C1. Specifically, we observed that FGFR2-C1-expressing cells grew poorly under anchorage-independent conditions in the absence of insulin. However, the FGFR2-C3-expressing cells thrived in soft agar in the presence or absence of insulin in their medium. Further, FGFR2C3 cells required a higher concentration of the FGFR small molecule kinase inhibitor PD173074 to abrogate their growth in soft agar, as compared to FGFR2-C1. An additional significant phenotypic difference between the isoforms was their ability to induce invasion of basement membrane. We found that the FGFR2-C3-expressing cells were able to invade at similar rates as breast cancer cells, whereas the FGFR2-C1-expressing cells were noninvasive (Moffa et al., 2004).

Signaling through FGFR is mediated by the adapter protein FGF receptor substrate 2 (FRS2). FRS2 is myristoylated, and thus constitutively membranebound (Kouhara et al., 1997). FRS2 is also bound to FGFR, regardless of receptor activation status (Xu et al., 1998). Once the receptor is activated by ligand binding, FRS2 is tyrosine phosphorylated by FGFR and serves as a docking site for other signaling molecules. Phosphorylated FRS2 binds to Grb2 and Shp2, leading to downstream activation of the PI3-K and MAPK pathways, respectively (Kouhara et al., 1997; Hadari et al., 1998). Our previous data indicated that the level of constitutively tyrosine phosphorylated FRS2 was substantially higher in the FGFR2-C3-expressing cells, as compared to FGFR2-C1 cells. The intensity of ligand-independent tyrosine phosphorylated FRS2 in the FGFR2-C3expressing cells was comparable to that observed in the SUM-52 breast cancer cells. In the FGFR2-C1 cells, however, the level of tyrosine phosphorylated FRS2 was low in the absence of KGF stimulation (Moffa et al., 2004).

In this work, we sought to examine further the signal transduction by the $\mathrm{C} 1$ and $\mathrm{C} 3$ containing isoforms of FGFR2-IIIb to understand better why the FGFR2-C3 isoform is more transforming of HME cells. Our results indicate that FGFR2-C3 is a constitutively active receptor tyrosine kinase, leading to strong and sustained activation of FRS2 and downstream signaling pathways. By contrast, the FGFR2-C1 isoform requires stimulation by KGF to achieve full activation. KGF-induced activation of FGFR2-C1 is transient, and results in a mobility shift of FRS2 that is not observed when this signaling molecule is activated by the C3 isoform of FGFR2. Site-directed mutagenesis of the FGFR2-C1 carboxyl terminus indicated that the removal of Tyr769 did not lead to more transforming activity in the FGFR2-C1 isoform. In addition to FGFR2 being highly overexpressed at the message and protein levels, these findings strongly suggest that the expression of alternatively spliced variants of FGFR2, particularly those containing the C3 carboxyl terminus, is required to fully induce the transformed phenotype of the SUM-52 breast cancer cells.

\section{MATERIALS AND METHODS Cell culture conditions}

H16N2 cells are an immortalized human mammary epithelial cell line that were immortalized by the human papillomavirus-16 genes E6, E7, and E5 (Band et al., 1990). The SUM-52 and H16N2 cells are cultured in Ham's F12 medium under serum-free conditions. The medium is supplemented with $0.1 \%$ BSA, $\quad 0.5 \mu \mathrm{g} / \mathrm{ml}$ fungizone, $5 \mu \mathrm{g} / \mathrm{ml}$ gentamicin, $5 \mathrm{mM}$ ethanolamine, $10 \mathrm{mM}$ HEPES, $5 \mu \mathrm{g} / \mathrm{ml}$ transferrin, $10 \mu \mathrm{M} \mathrm{T}_{3}$, $50 \mu \mathrm{M}$ selenium, $1 \mu \mathrm{g} / \mathrm{ml}$ hydrocortisone, and $5 \mu \mathrm{g} / \mathrm{ml}$ insulin. Vector-control H16N2 cells also grow in the presence of $10 \mathrm{ng}$ ml EGF. Transduced H16N2 cells grow in G418-supplemented media to maintain expression of the transgene. All cell culture reagents were obtained from Sigma Chemical Co. (St. Louis, MO).

\section{Site-directed mutagenesis}

Mutagenesis of FGFR2-C1 to FGFR2-C1-Y769F was performed using the Stratagene QuikChange XL Site-Directed Mutagenesis Kit (Stratagene, La Jolla, CA), according to the manufacturer's instructions. In short, the "tac" codon at base 2,900 was mutated to "ttc" in order to substitute a Phe for a Tyr at amino acid 769. Primers were designed which amplified the insert-containing plasmid ( $\mathrm{pNG}$ ), and introduced the appropriate mutation. (Forward: TCACAACCAATGAGGAAttcTTGGACCTCAGCCAA and reverse: TTGGCTGAGGTCCAAgaaTTCCTCATTGGTTGTGA, with the target codon denoted in bold, lower-case.) The kit provides PfuTurbo DNA polymerase, which replicates both plasmid strands with high fidelity. The plasmid is then transformed into XL10-Gold ultracompetent cells (provided with the kit). We next performed minipreps (Qiagen, Valencia, CA) and the prepared DNA was sent for sequencing to the Wayne State University DNA Sequencing Core. (Sequencing primers were forward: ATGGATAAGCCAGCCAACTG and reverse: GAGGAAGGCATGGTTCGTAA, with each approximately $100 \mathrm{bp}$ from the mutation.) Sequencing verified introduction of the desired mutation.

\section{Development of FGFR2-C1-Y769F-expressing cell lines}

The FGFR2-C1-Y769F-expressing pNG plasmid was transfected into $\phi \mathrm{nx}-\mathrm{A}$ viral packaging cells. $\phi \mathrm{nx}$-A cells were plated at $2 \times 10^{6}$ cells $/ 60-\mathrm{mm}$ dish 1 day prior to transfection. Cells were transfected using the calcium phosphate method. Briefly, $\phi n x-A$ cells were incubated in 10\% Dulbecco's modified eagle medium with $50 \mu \mathrm{M}$ chloroquine for $5 \mathrm{~min}$. A reaction mix of $5 \mu \mathrm{g}$ DNA, $1 \times$ HEPES-buffered saline, and $\mathrm{CaCl}_{2}$ (JT Baker, Phillipsburg, NJ) was added to $\phi$ nx-A dishes. Media were changed $8 \mathrm{~h}$ after transfection, and again $24 \mathrm{~h}$ later. Fortyeight hours after transfection, viral particles were collected, centrifuged, and filtered. Virus was frozen at $-80^{\circ} \mathrm{C}$, then the viral particles collected the following day $(72 \mathrm{~h}$ post-transfection) were used to infect H16N2 human mammary epithelial cells.

H16N2 cells were grown to approximately $30-50 \%$ confluence and infected with prepared viral particles. In order to aid in fusion of cell membranes, cells were also treated with $5 \mu \mathrm{g} / \mathrm{ml}$ polybrene. Media were changed on the $\mathrm{H} 16 \mathrm{~N} 2$ cells $24 \mathrm{~h}$ following infection. After $48 \mathrm{~h}$, cells were now fed with selection media, including G418. One 60-mm dish was grown in the presence of EGF and one was grown without EGF, in order to apply selection pressure for cells that have taken up the mutant version of FGFR2-C1.

\section{RT-PCR verification of FGFR2-C1-Y769F expression}

Total RNA was isolated using the Qiagen RNeasy kit. RNA was converted into $\mathrm{cDNA}$ via a reverse transcription reaction with random hexamer primers, using the SuperScript FirstStrand Synthesis System for RT-PCR (Invitrogen, Carlsbad, CA). Product was then used for amplification of FGFR2 using gene specific primers to the conserved transmembrane domain portion of the receptor (forward: AGCAAGCGCCTGGAAGAGAAAA and reverse: GGCTTATCCATTCTGTGTCCTTC). The AccuPrime Pfx (Invitrogen) protocol was followed, using 35 cycles of $94^{\circ} \mathrm{C}$ for $30 \mathrm{sec}, 56^{\circ} \mathrm{C}$ for $1 \mathrm{~min}$, and $72^{\circ} \mathrm{C}$ for $1.5 \mathrm{~min}$, 
with a final extension at $72^{\circ} \mathrm{C}$ for $10 \mathrm{~min}$. RT-PCR product was then run in a $0.8 \%$ agarose gel for analysis.

\section{FGFR2 Western blots}

Cells were rinsed twice with ice cold $1 \times \mathrm{PBS}$ and then lysed on ice with a buffer consisting of $50 \mathrm{mM}$ Tris-HC1, $\mathrm{pH} 8.5$, $150 \mathrm{mM} \mathrm{NaCl}, 1 \%$ Nonidet P-40 (ICN Biomedical, Inc., Aurora, $\mathrm{OH}$ ), and $5 \mathrm{mM}$ EDTA, supplemented with $5 \mathrm{mM}$ sodium orthovanadate, $10 \mathrm{mM}$ sodium pyrophosphate, $50 \mu \mathrm{g} / \mathrm{ml}$ phenylmethsulfonyl fluoride (PMSF), $20 \mu \mathrm{g} / \mathrm{ml}$ aprotinin, and $10 \mu \mathrm{g} / \mathrm{ml}$ leupeptin. Lysates were spun at $20,800 \mathrm{~g}$ at $4^{\circ} \mathrm{C}$ for $10 \mathrm{~min}$ and then analyzed for protein using a modified Lowry's method. For detection of FGFR2-C1 mutant and wild type variants, $100 \mu \mathrm{g}$ of whole cell lysates were resolved in 7.5\% polyacrylamide gels, transferred to PVDF membrane (Millipore Corporation, Bedford, MA) and probed with an antiFGFR2 antibody (Santa Cruz Biotechnology, Inc., Santa Cruz, CA). This antibody is specific for the $\mathrm{C} 1$ carboxyl terminus of FGFR2, and therefore does not detect the FGFR2-C3 isoform. The blots were developed with ECL Western Blotting Substrate (Pierce, Rockford, IL) and exposed to film.

\section{Assessment of monolayer growth}

Cells were plated into 6 -well dishes at $3.5 \times 10^{4}$ cells/well, in serum-free media in the presence of insulin and the absence of EGF. Cells were fed every other day with the appropriate media. The concentration of FGFR inhibitor PD173074 was $1 \mu \mathrm{M}$ and KGF-treated cells were treated with $25 \mathrm{ng} / \mathrm{ml} \mathrm{KGF}$ (Sigma) plus $2 \mu \mathrm{g} / \mathrm{ml}$ heparin. Plating efficiency cell counts were determined $24 \mathrm{~h}$ after originally plating the cells, and this was considered day 0 for the experimental groups. The number of cells was determined by counting isolated nuclei with a
Coulter Counter at day 0, 3, and 7 after plating. All experiments were done in duplicate, twice.

\section{Growth in soft agar}

A bottom layer of 1:1 Ham's F-12 serum free medium and $1 \%$ agarose was poured and allowed to solidify. A $1 \mathrm{ml}$ suspension of $1 \times 10^{5}$ cells in a $0.3 \%$ agarose solution was plated into 6 -well dishes and fed every other day by adding $1 \mathrm{ml}$ of medium on top of the soft agar. Cells were maintained in serum-free medium, in the presence of insulin and the absence of EGF. After 2 weeks, excess medium was carefully removed from the wells, and $1 \mathrm{mg} / \mathrm{ml}$ solution of $p$-iodonitrotetrazolium violet (Sigma) was added to the wells overnight to stain for viable cells. Stained viable cell colonies were photographed the following day.

\section{FRS2 Western blots}

Cells were treated for $10 \mathrm{~min}$ in the presence or absence of $25 \mathrm{ng} / \mathrm{ml} \mathrm{KGF}$ (Sigma) $+2 \mu \mathrm{g} / \mathrm{ml}$ heparin. Cells were lysed as described above, and $1 \mathrm{mg}$ whole cell lysate was affinity precipitated with $\mathrm{p} 13^{\mathrm{suc} 1}$-agarose-conjugated beads (Upstate, Lake Placid, NY). Samples were run in an SDS-PAGE gel and blotted onto PVDF membrane. The FRS2 antibody was purchased from Santa Cruz, and the anti-phosphotyrosine antibody (4G10) is from Upstate. These blots were developed via ECL. All Western blots are representative of at least two different experiments.

For Figures 1 and 6, cells were exposed to KGF and heparin for $10 \mathrm{~min}$, then the plates were rinsed and fresh media were added, without KGF. The cells were lysed following the indicated number of hours after removal of KGF, and the Western blot was performed as described above. Quantification

$\mathbf{A}$

Affinity precipitate: $\mathrm{p} 13^{\text {suc }}$-agarose beads Blot: $\alpha$-phosphotyrosine

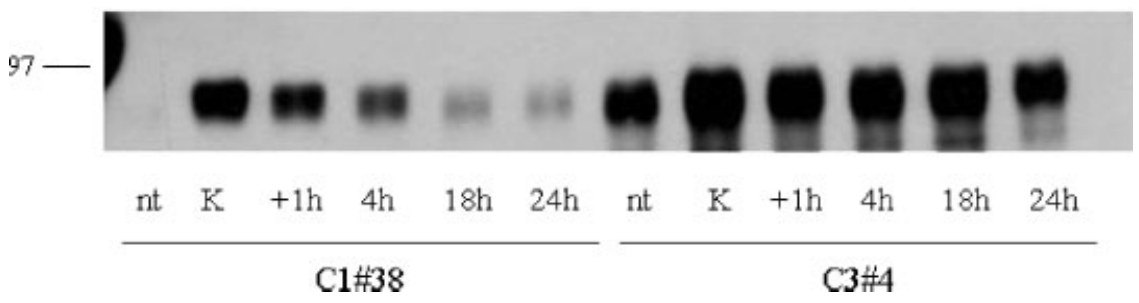

B

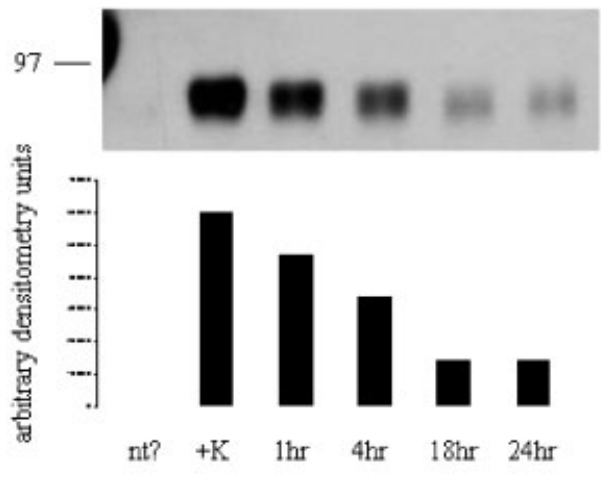

C1\#38

Fig. 1. Time course of FRS2 tyrosine phosphorylation after KGF stimulation of FGFR2-C1 and C3 cells. A: FGFR2-C1 and C3 cells were either not treated (nt), or treated with $25 \mathrm{ng} / \mathrm{ml} \mathrm{KGF}$ and $10 \mu \mathrm{g} /$ $\mathrm{ml}$ heparin for $10 \mathrm{~min}(\mathrm{~K})$. The remaining samples were treated with KGF for $10 \mathrm{~min}$, rinsed, and then lysed after the indicated number of hours. FRS2 was affinity precipitated with $\mathrm{p} 13^{\text {suc1 }}$-agarose-conjugated

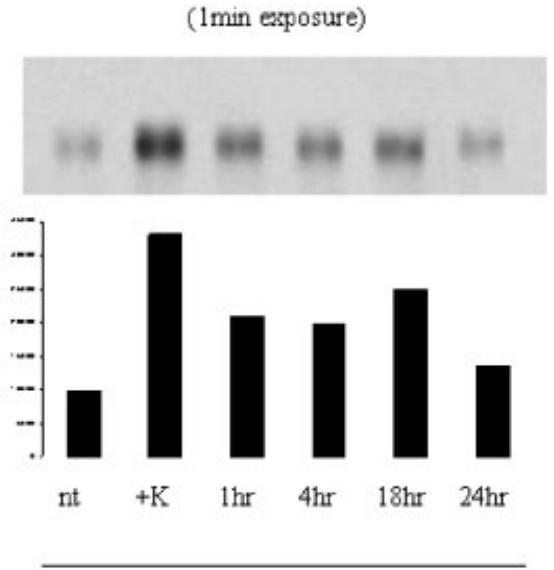

\section{C3\#4}

beads, and the Western blot was probed with a phosphotyrosine antibody. B: This figure shows differently exposed blots for each cell line. The FGFR2-C1 half of the blot was exposed for $10 \mathrm{~min}$ and the C3 for $1 \mathrm{~min}$. Quantification of the intensities of the data shown is depicted in the graph below each blot. 
was performed utilizing Bio-Rad (Hercules, CA) Quantity One software.

Figure 7 shows cells treated with or without KGF and heparin for $10 \mathrm{~min}$. Hundred micrograms of whole cell lysate was run directly into an SDS-PAGE gel. These blots were probed with phosphospecific FRS2 antibodies, purchased from Cell Signaling (Beverly, MA), that recognize pTyr196 and pTyr436 of FRS2.

\section{Erk Western blots}

Cells were treated $24 \mathrm{~h}$ in the presence or absence of $1 \mu \mathrm{M}$ PD173074, or $10 \mathrm{~min}$ in the presence or absence of $25 \mathrm{ng} / \mathrm{ml}$ $\mathrm{KGF}+2 \mu \mathrm{g} / \mathrm{ml}$ heparin. Cells were lysed as described above, and $100 \mu \mathrm{g}$ whole cell lysate was run in an SDS-PAGE gel and blotted onto PVDF membrane. Antibodies used were anti-Erk1 and phospho-Erk (Santa Cruz). These blots were also developed via ECL.

\section{Phosphothreonine FRS2 analysis}

Cells were treated with the MAPK inhibitor U0126 (Promega, Madison, WI) at $5 \mu \mathrm{M}$ for $30 \mathrm{~min}$, or $10 \mathrm{~min}$ with KGF, and heparin. We have previously observed that $30 \mathrm{~min}$ of $5 \mu \mathrm{M}$ U0126 treatment abrogates phosphorylation of Erk, indicating inhibition of MEK (data not shown). Cells were lysed as described above, and $1 \mathrm{mg}$ of whole cell lysate was immunoprecipitated with the FRS2 antibody (Santa Cruz). Samples were run in an SDS-PAGE gel and blotted onto PVDF membrane. The blots were probed with antibodies against phosphotyrosine (Upstate) or phosphothreonine followed by a proline (Cell Signaling).

\section{RESULTS}

To investigate the transforming potential of alternatively spliced FGFR2 variants expressed by the SUM-52 breast cancer cells, we previously stably overexpressed the full-length FGFR2-C1 and FGFR2-C3 isoforms into H16N2 human mammary epithelial cells.

\section{FRS2 activation followed by KGF stimulation}

As our early work showed that FRS2 is constitutively highly tyrosine phosphorylated in the FGFR2-C3expressing H16N2 cells, and only weakly phosphorylated in the FGFR2-C1 cells, we further analyzed the activation of this signaling molecule. We have observed previously that KGF induced an increased tyrosine phosphorylation and upward electrophoretic mobility shift of FRS2 in the FGFR2-C1-expressing cells (Moffa et al., 2004). This result was observed with as brief as a 10-min exposure of the FGFR2-C1 cells to KGF, as well as continuous KGF stimulation overnight (approximately $14 \mathrm{~h}$ ). However, it was unclear how long the FGFR2 isoforms remained activated after stimulation with exogenous ligand.

In order to address this question, FGFR2-C1 and C3 cells were treated with KGF for $10 \mathrm{~min}$, the media changed, and the cells lysed after varying amounts of time. FRS2 was affinity precipitated from cell lysates with p $13^{\text {suc1 }}$-agarose-conjugated beads, and levels of FRS2 tyrosine phosphorylation were assessed (Fig. 1A). As we have observed previously, the basal level of tyrosine phosphorylated FRS2 was much higher in the FGFR2-C3-expressing cells than FGFR2-C1. Clearly, both cell lines responded to KGF stimulation, showing increases in the tyrosine phosphorylation of FRS2 following $10 \mathrm{~min}$ exposure to KGF. Figure 1B shows differently exposed films from the same blot, and quantification of band intensity. In order to appropriately visualize and compare the tyrosine phosphorylated levels of FRS2 in the FGFR2-C1 versus C3 cells, the samples from the FGFR2-C3-expressing cells were exposed to film for one-tenth the time as the $\mathrm{C} 1$ samples.
Furthermore, even with the prolonged exposure time, the basal level of tyrosine phosphoryated FRS2 in the FGFR2-C1-expressing cells was undetectable, and thus not quantifiable. Therefore, these data show that FRS2 is constitutively tyrosine phosphorylated to high levels in the FGFR2-C3 cells, regardless of the presence of ligand. Even though KGF induces an enhanced intensity of tyrosine phosphorylated FRS2 that is subject to downregulation upon removal of ligand, the basal levels remain high in the presence or absence of KGF. In contrast, the FGFR2-C1 cells were dependent on continuous KGF stimulation to achieve high levels of FRS2 tyrosine phosphorylation.

\section{Site-directed mutation of FGFR2-C1 carboxyl terminus}

Due to our observations of the dramatically different transforming potential of FGFR2-C3 versus FGFR2-C1, we next sought to analyze the structural differences between the proteins. A logical target was Tyr769, as this potentially important signaling residue is present on the FGFR2-C1 carboxyl terminus, and absent from C3 (Itoh et al., 1994). In addition to binding PLC $\gamma$, Cross et al. have shown that phosphorylated Tyr766 on FGFR1 binds to the Shb adapter protein, contributing to MAPK activation (Mohammadi et al., 1991; Cross et al., 2002). Further, data from Lax et al. (2002) indicated that MAPK threonine phosphorylates FRS2, which serves as a negative feedback loop and leads to a decrease in tyrosine phosphorylation of FRS2. Since only FGFR2-C1 has the appropriate residue to bind to $\mathrm{Shb}$, we hypothesized that this mechanism of negative regulation of FRS2 via MAPK might be responsible for the controlled signaling in the FGFR2-C1-expressing cells, as compared to the robust constitutive level of tyrosine phosphorylated FRS2 observed in cells expressing the FGFR2-C3 isoform lacking Tyr769.

To determine the importance of Tyr769 on the C1 carboxyl terminus, we performed site-directed mutagenesis of tyrosine to phenylalanine. FGFR2-C1-Y769F was created by PCR-based site-directed mutagenesis, and verified by DNA sequencing. The mutant was introduced into H16N2 human mammary epithelial cells via a stable bicistronic retroviral infection using the same pNG vector as the wild type FGFR2-C1 and C3 genes. Transduced cells were selected for resistance to G418, and grown in serum-free medium supplemented with insulin, in the presence or absence of EGF. Expression of C1-Y769F message (tested via RT-PCR) and protein (Western blotting) in the H16N2 cells was validated and the data are shown in Figure 2A, B.

The C1-Y769F cells originally grown in the presence of EGF were either propagated as individual clones, or as a polyclonal population. YF\#7 is representative of a highly-expressing clone, and YFpc cells are the original polyclonal population. Another clone, YF\#8, was unable to grow in the absence of EGF and, without this selection pressure, eventually lost C1-Y769F expression entirely, as shown in Figure 2B. YF-EGF cells were initially selected directly for EGF-independent growth, and were sustained as a polyclonal population. When these cells were originally being selected, they grew more slowly than the cells grown in the presence of EGF. At the present time, all C1-Y769F-expressing cells are growing robustly in the absence of EGF.

As growth factor independence is a hallmark of transformation, we assessed the ability of FGFR2-C1Y769F cells to grow in medium lacking EGF. This is the condition under which the FGFR2-C1 wild type, 
$\mathbf{A}$

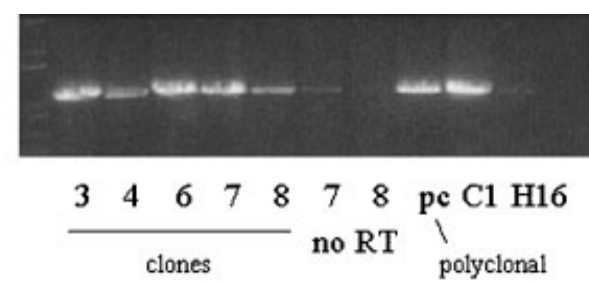

B

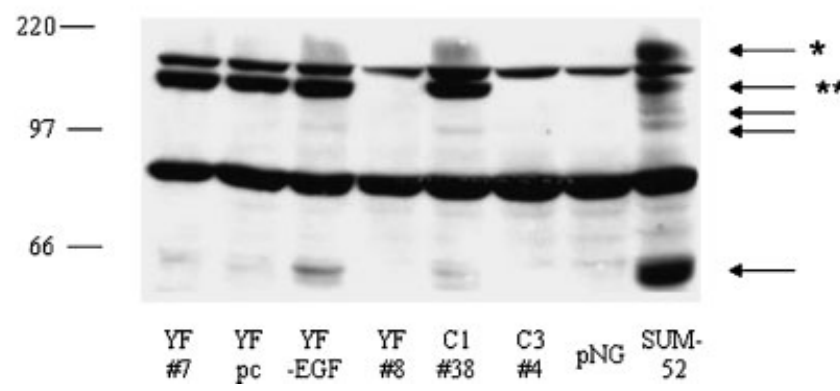

Fig. 2. Expression of FGFR2-C1-Y769F into H16N2 cells. A: RT-PCR analysis of message levels of C1-Y769F. Numbered samples are individual clones, and "pc" denotes a polyclonal population. "No RT" samples were run without reverse transcriptase enzyme. C1 represents wild type FGFR2-C1, and H16 are untransduced H16N2 cells. B: This Western blot is whole cell lysate of the indicated samples, blotted with an FGFR2 antibody. The antibody specifically recognizes the FGFR2-C1 carboxyl terminus, so the FGFR2-C3 lane is blank. The ** represents the full-length FGFR2-C1 isoform, and * is the glycosylated version of that isoform. The other arrows denote the additional C1-containing FGFR2 isoforms expressed by the SUM-52 cells. The YF\#7 and YF\#8 cell lines were cloned from H16N2 cells infected with FGFR2-C1-Y769F, and originally cultured in the presence of EGF. Clone YF\#8 was unable to survive in the absence of EGF, and therefore did not show expression of FGFR2-C1-Y769F. YFpc cells are a polyclonal population of cells originally cultured with EGF. YF-EGF cells were initially cultured in the absence of EGF. Currently, all FGFR2-C1-Y769F-expressing cells are cultured in the absence of EGF.

FGFR2-C3, and SUM-52 cells routinely grow, while H16N2 cells normally depend on EGF for growth. We analyzed EGF-independent growth of cells highly expressing the $\mathrm{C} 1-\mathrm{Y} 769 \mathrm{~F}$ clone (YF\#7), and compared them to FGFR2-C1 wild type expressing cells, and cells expressing the pNG empty vector. The growth curve in Figure 3A shows that the C1-Y769F-expressing cells grew at a similar rate in the absence of EGF as cells expressing wild type FGFR2-C1. The C1-Y769F cells grew and survived without EGF, whereas the pNG empty vector expressing H16N2 cells did not, indicating that this FGFR2 mutant induced growth factor independent proliferation and survival, like the wild type FGFR2-C1.

We next treated cells with the small molecule FGFR kinase inhibitor PD173074 and assessed their growth capacity. We observed that growth of the C1-Y769Fexpressing cells in EGF-free medium was blocked by the FGFR inhibitor (Fig. 3B). The FGFR2-C1 cells' growth was also inhibited by PD173074, and the pNG empty vector expressing cells were unaffected. This indicates that the FGFR2-C1-Y769F and FGFR2-C1 wild type expressing cells are dependent upon FGFR2 activity to grow in the absence of EGF. Finally, we assessed the C1Y769F cells' responsiveness to exogenous KGF, since $\mathrm{KGF}$ is a natural ligand for FGFR2-IIIb. The data in Figure 3C indicate that the C1-Y769F and FGFR2-C1 wild type cells displayed a slight growth advantage in the presence of KGF in the media, in the absence of EGF. However, the SUM-52 breast cancer cells and H16N2 cells overexpressing FGFR2 required neither EGF nor KGF for growth or survival, as evidenced by their routine culture in serum-free medium lacking exogenous EGF or KGF. In addition, we have determined by ELISA that these cells do not secrete KGF, ruling out an autocrine activation of FGFR2 by KGF (data not shown). However, FGFR2b also binds FGF1, 3, 10, and 22, allowing the possibility of an autocrine loop with one of these other ligands. Overall, our phenotype data suggest that H16N2 cells overexpressing FGFR2-C1-Y769F, wild type FGFR2-C1, or C3 proliferate in the absence of exogenous EGF or KGF in monolayer.

To study further the transformation of the FGFR2C1-Y769F mutant expressing cells, we tested their ability to grow under anchorage-independent conditions. Cells were plated in soft agar, and cultured in media containing insulin and lacking EGF. Viable cell colonies were stained and photographed after 2 weeks. Figure 4 shows that the C1-Y769F-expressing cells were able to survive and proliferate under anchorage-independent conditions in the absence of EGF, similarly to the wild type FGFR2-C1. In contrast, the pNG empty vector expressing cells did not survive in soft agar in EGF-free medium. These data suggest that overexpression of the mutant C1-Y769F version of FGFR2 induces transformed phenotypes in human mammary epithelial cells, comparable to FGFR2-C1 wild type.

\section{Signal transduction by FGFR2-C1-Y769F}

Next, we performed Western blots examining the phosphorylation status of FRS2 in the FGFR2-C1Y769F-expressing cells, as compared to wild type FGFR2-C1, C3, and SUM-52 cells. FRS2 was affinity precipitated from cell lysates, and the levels of FRS2 protein and tyrosine phosphorylation were assessed. Figure 5 shows that basal FRS2 tyrosine phosphorylation was low in the C1-Y769F mutant expressing cells, similar to the cells expressing wild type FGFR2-C1. In contrast, constitutive tyrosine phosphorylation of FRS2 in the FGFR2-C3-expressing cells was much higher. Upon KGF stimulation, the tyrosine phosphorylation and the apparent molecular weight of FRS2 increased in the FGFR2-C1-Y769F and C1 wild type cells. Tyrosine phosphorylation of FRS2 in the FGFR2-C3-expressing cells was also increased in the presence of $\mathrm{KGF}$, with the basal level already high. The pNG empty vector expressing H16N2 cells showed no tyrosine phosphorylation of FRS2 in the presence or absence of KGF, as expected. SUM-52 breast cancer cells displayed robust, constitutive tyrosine phosphorylation of FRS2 with or without KGF. These results suggested that signaling from the mutant Y769F variant of FGFR2-C1 did not mimic the signaling capacity of the FGFR2-C3 isoform. Instead, both mutant C1-Y769F and wild type FGFR2C1-expressing cells depended on KGF to induce a high level of tyrosine phosphorylation of FRS2, as compared to the high constitutive level seen in the FGFR2-C3expressing cells.

We next sought to analyze the time course of FRS2 deactivation after withdrawal of KGF in the FGFR2-C1Y769F-expressing cells. This experiment was performed in an identical manner to that shown in Figure 1, with the cells exposed to KGF for $10 \mathrm{~min}$, then lysed after varied amounts of time and FRS2 tyrosine phosphorylation studied. Figure 6 shows that, in parallel to wild type FGFR2-C1-expressing cells, the levels of FRS2 phosphotyrosine in the untreated mutant cells were undetectable, and they displayed a robust response to KGF stimulation. Once again, this result more closely mimics 
that observed in the FGFR2-C1-expressing cells, with FGFR2-C1-Y769F cells not able to express the high, sustained level of FRS2 tyrosine phosphorylation detected in the FGFR2-C3 cells in the absence of ligand. These results also confirm and extend our observations that activation of both mutant and wild type FGFR2-C1 isoforms results in an FRS2 mobility shift upon KGF stimulation that is associated with a rapid loss of tyrosine phosphorylated FRS2.

Tyrosine phosphorylated FRS2 is known to interact directly with Grb2 and Shp2, leading to downstream activation of the PI3-K and MAPK pathways. Phosphotyrosines 196 and 436 on FRS2 are binding sites for Grb2 and Shp2, respectively (Hadari et al., 1998; Kouhara et al., 1997). Western blots of whole cell lysate protein indicate that both tyrosines 196 and 436 were constitutively phosphorylated in $\mathrm{H} 16 \mathrm{~N} 2$ cells expressing the FGFR2-C1-Y769F mutant, wild type FGFR2-C1, and C3, as well as the SUM-52 breast cancer cells (Fig. 7). Neither of these FRS2 tyrosines was phosphorylated in H16N2 cells expressing the pNG empty vector, as expected. The basal levels of tyrosine phosphorylation of Tyr196 and 436 in the FGFR2-C3-expressing cells and the SUM-52 cells were notably higher than the cells expressing either wild type or mutant FGFR2-C1. Stimulation of the FGFR2-C1-Y769F and wild type C1 cells with KGF led to a dramatic increase in phosphotyrosine intensity, as well as an apparent molecular

A
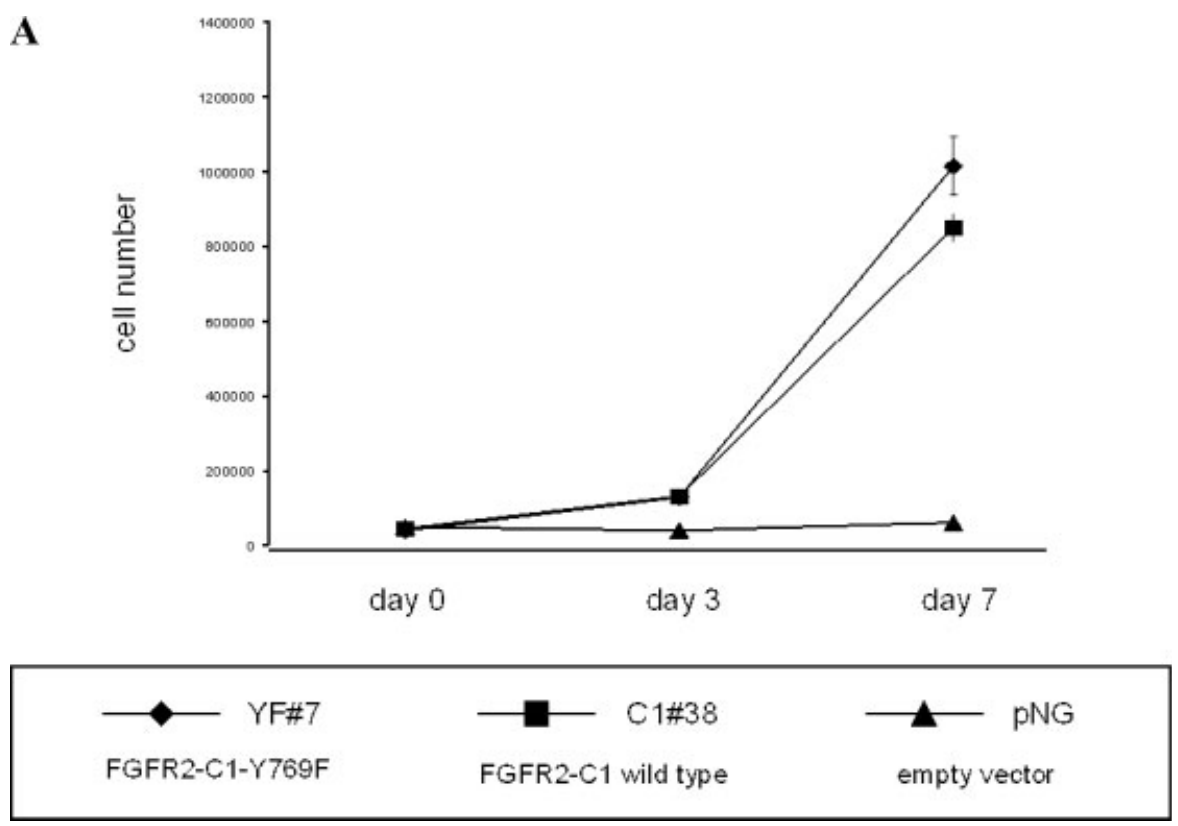

B
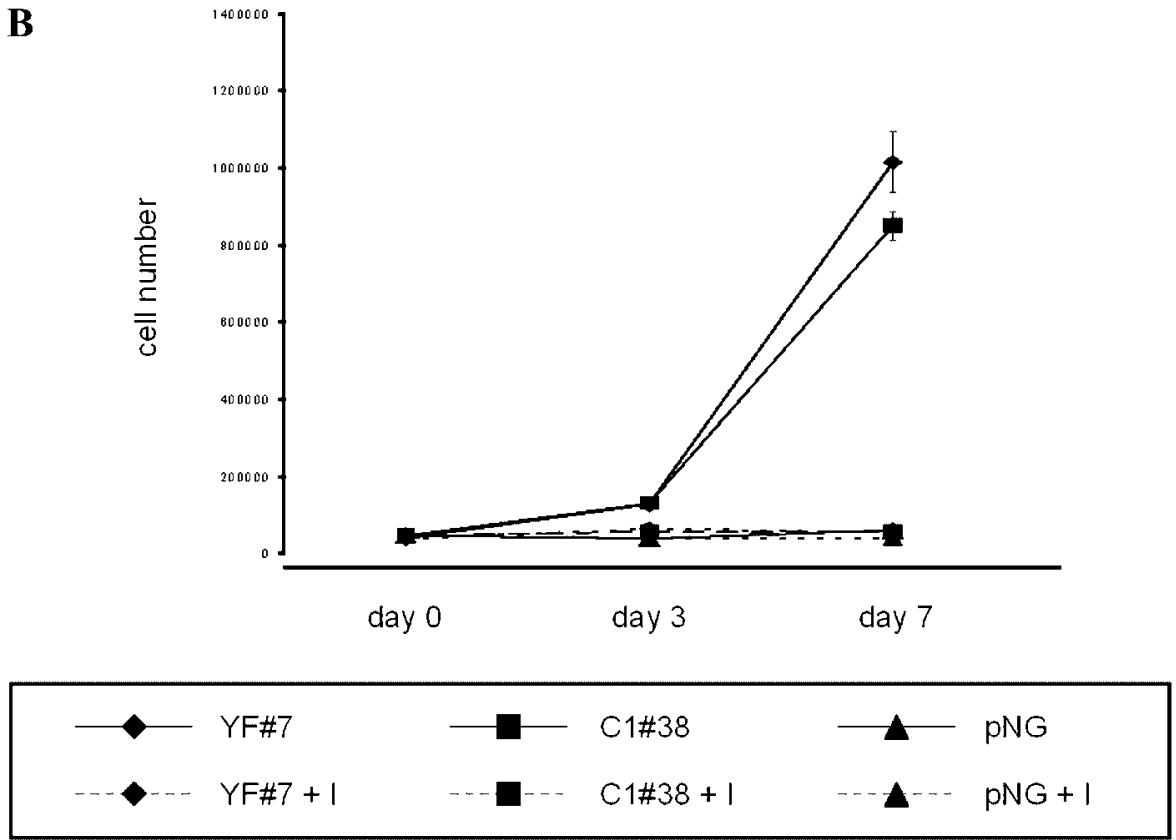

Fig. 3. Growth curves of FGFR2-C1-Y769F cells. Cells were plated in 6 -well dishes at $5 \times 10^{3}$ cells/well. Nuclei were isolated and automatically counted after the indicated number of days. A: Ability of FGFR2$\mathrm{C} 1-\mathrm{Y} 769 \mathrm{~F}, \mathrm{C} 1$ wild type, and empty vector (pNG) expressing cells to grow in the absence of EGF. B: Growth curve of cells in the presence or absence of $1 \mu \mathrm{M}$ FGFR kinase inhibitor PD173074 (I). Please note that all three treated cell lines, and the untreated pNG cells, were unable to grow under these conditions, and thus the data overlap. Cells were fed and treated every other day. C: Growth curve analysis of FGFR2 C1-Y769F, C1 wild type, and pNG cells in the presence or absence of $25 \mathrm{ng} / \mathrm{ml} \mathrm{KGF}$ and $10 \mu \mathrm{g} / \mathrm{ml}$ heparin $(\mathrm{KGF})$. Cells were fed and treated every other day. 


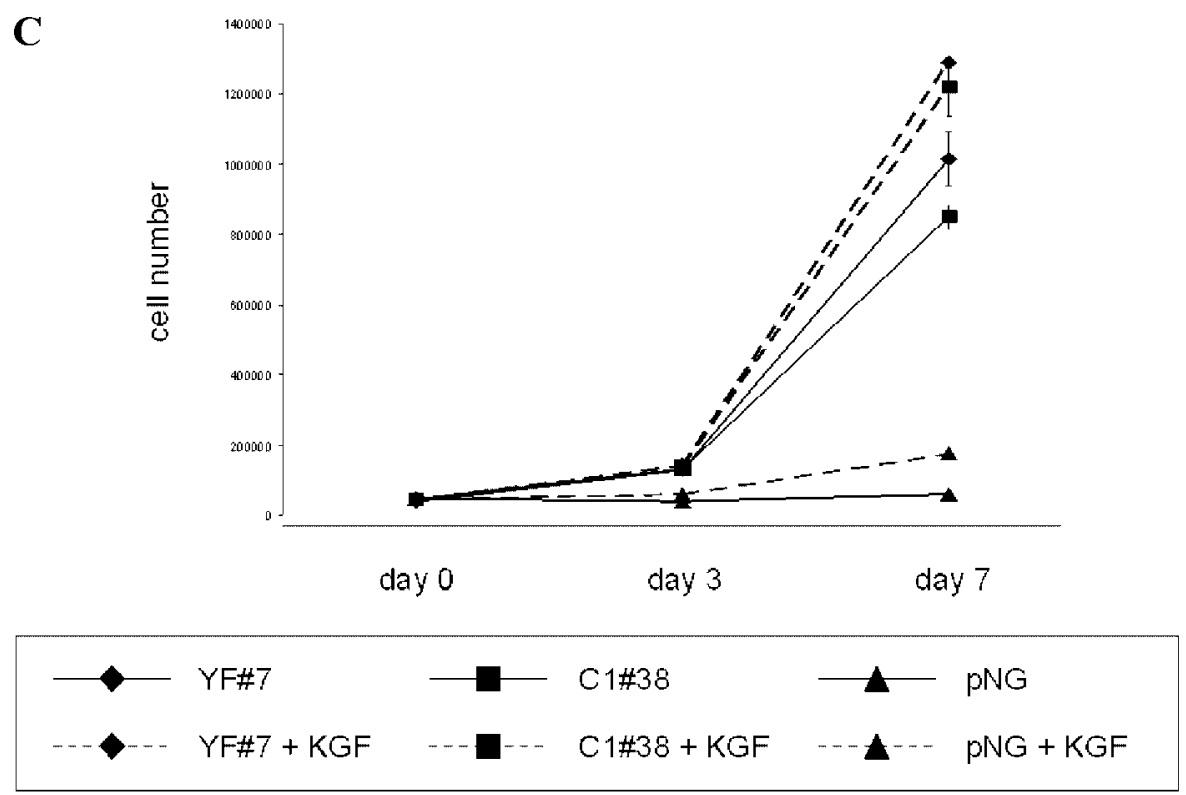

Fig. 3. (Continued)

weight increase of the FRS2 protein, depicted in both phospho-specific blots shown in Figure 7. These data indicated that the basal level of tyrosine phosphorylated FRS2 leading to activation of major downstream pathways was dramatically higher in FGFR2-C3-expressing cells, as compared to mutant or wild type FGFR2-C1. In all of the FGFR2 overexpressing cells tested, the FGFR kinase inhibitor PD173074 completely abrogated the phosphorylation of both sites on FRS2, indicating that FGFR2 activity was required for FRS2 activation at multiple sites (data not shown).

To study signaling downstream from FGFR2/FRS2, we next analyzed activation of the MAPK pathway. Phosphorylation of Erk- 1 and 2 serves as an indication of MAPK activity. Cells were untreated, treated for $24 \mathrm{~h}$ with the FGFR kinase inhibitor PD173074, or treated for $10 \mathrm{~min}$ with KGF. Cells were then lysed, and subjected to Western blotting for Erk and phospho-
Erk. Data in Figure 8 suggest that Erk phosphorylation is enhanced in the FGFR2-C1-Y769F and wild type C1expressing cells in the presence of KGF. Basal Erk phosphorylation levels appear higher in the FGFR2-C1Y769F-expressing cells as compared to wild type FGFR2-C1 cells. In this case, Erk activation by FGFR2-C1-Y769F more closely mimics that observed in the FGFR2-C3-expressing cells. However, both the mutant and wild type FGFR2-C1-expressing cells respond to KGF stimulation, whereas levels of Erk phosphorylation in the FGFR2-C3-expressing cells appear unchanged.

Erk phosphorylation in the FGFR2 overexpressing H16N2 cells was only partially inhibited by the FGFR kinase inhibitor, as we have observed previously (Moffa et al., 2004). The residual levels of Erk phosphorylation upon FGFR inhibition may be attributable to insulinmediated activity. The vector control pNG cells

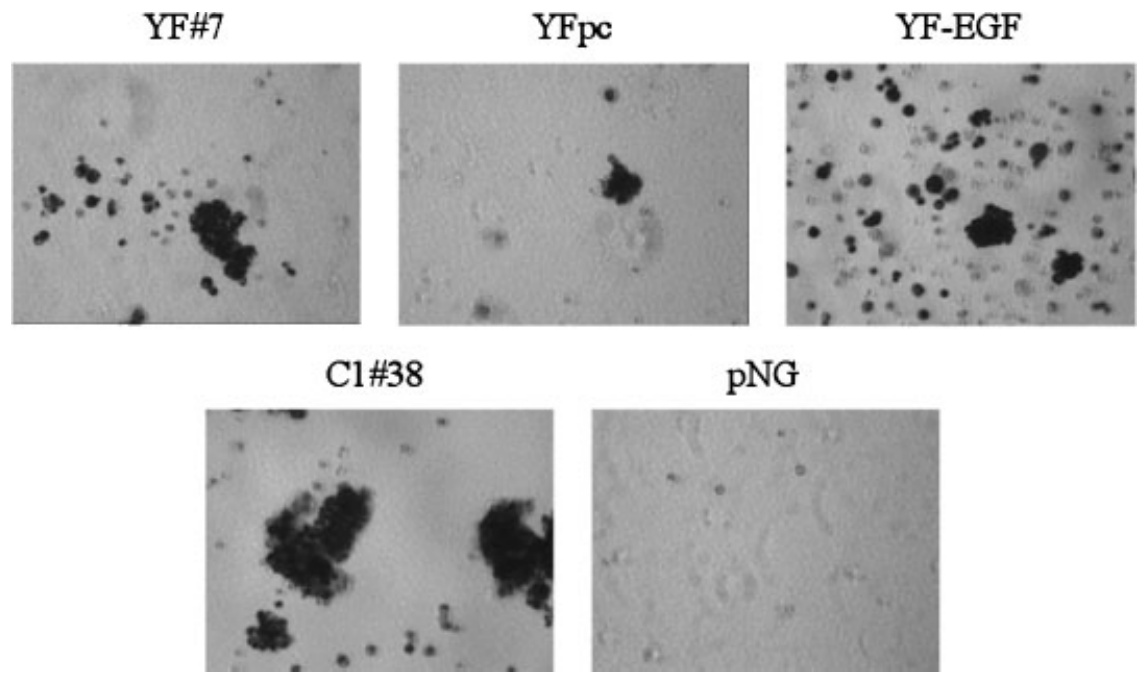

Fig. 4. Anchorage-independent growth of FGFR2-C1-Y769F and wild type FGFR2-C1-expressing cells. Cells were grown for 2 weeks in soft agar as described in Materials and Methods. Samples were maintained in serum-free media, in the presence of insulin and absence of EGF. Viable colonies were stained and photographed. 
FRS2 affinity precipitate: p13 suc1-agarose beads Blot: $\alpha$-phosphotyrosine
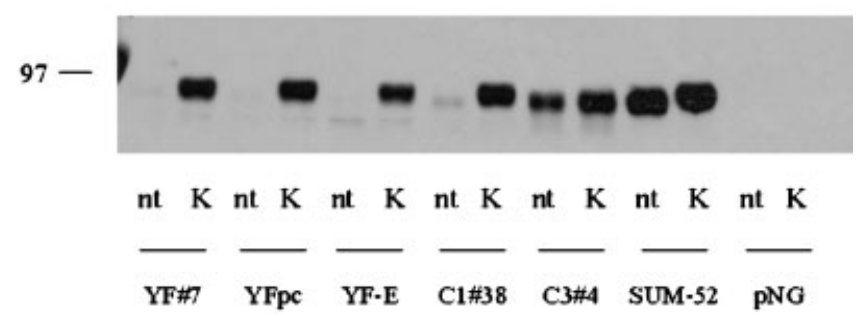

Blot: $\alpha$-FRS2

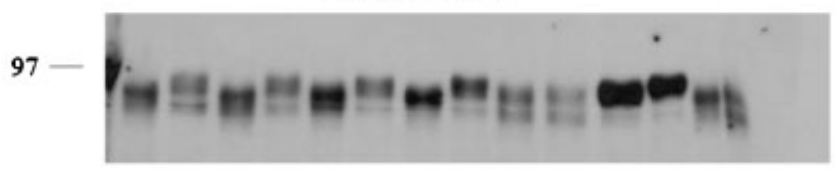

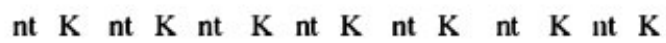

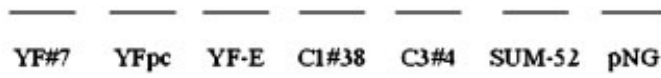

Fig. 5. Tyrosine phosphorylation of FRS2 in the FGFR2-C1-Y769F, $\mathrm{C} 1$ wild type, C3, pNG expressing cells, and SUM-52 cells. Samples labeled "nt" are not treated, and "K" lanes were treated with KGF and heparin for $10 \mathrm{~min}$. FRS2 was affinity precipitated and blots were probed for phosphotyrosine or FRS2.

expressed a low level of constitutively phosphorylated Erk, since these cells are cultured in the presence of both insulin and EGF, whereas the FGFR2 overexpressing cells are all cultured in the absence of EGF. Erk phosphorylation in the pNG-expressing cells was unaffected by KGF stimulation, nor was it blocked by FGFR kinase inhibition (Fig. 8).

Together, these results indicate that the FGFR2-C1 isoform with Tyr769 mutated to a phenylalanine is not a more transforming variant than the wild type FGFR2C1. Based on the literature, we assume that Tyr769, homologous to Tyr766 on FGFR1, solely mediates the interaction between the FGFR2 molecule and the signaling proteins PLC $\gamma$ and Shb. However, the possibility remains that either FGFR2-C1 or C3 may otherwise interact with PLC $\gamma$ or Shb, independent of Tyr769. Overall, our data suggest that preventing interactions between FGFR2-C1 Tyr769 and either PLC $\gamma$ or Shb did not create a receptor that mimics the signaling capacity of the FGFR2-C3 isoform.

\section{Threonine phosphorylation of FRS2}

Finally, we sought to analyze the phosphothreonine status of FRS2 in the H16N2 cells overexpressing the FGFR2-C1 or C3 isoform, and the SUM-52 breast cancer cells. Lax et al. (2002) have reported that there are eight threonine residues on FRS2 that are sites for phosphorylation by MAPK. Since we observed a significant upward electrophoretic mobility shift of FRS2 upon stimulation of FGFR2-C1-expressing cells with the KGF ligand, we hypothesized that this apparent molecular weight increase could be due to threonine phosphorylation of FRS2.

We utilized the MAPK inhibitor U0126 to assess the role of MAPK signaling in the threonine phosphorylation of FRS2 in our cells. We observed that the p13 $3^{\text {suc1 }}$. agarose-conjugated beads were unable to pull down FRS2 in the presence of U0126, suggesting that the association between FRS2 and p13 ${ }^{\text {suci }}$ was dependent upon serine/threonine phosphorylation of FRS2 (data not shown). Therefore, we immunoprecipitated FRS2 with an anti-FRS2 antibody and blotted with an antibody specific for the sites threonine phosphorylated by MAPK (pThr-Pro), as well as for tyrosine phosphorylation. Cells were untreated, treated with the U0126 MAPK inhibitor for $30 \mathrm{~min}$, or treated with KGF for $10 \mathrm{~min}$. The phosphothreonine blot in Figure 9 indicates that MAPK was indeed responsible for threonine phosphorylating FRS2 in our cells, as the phosphorylation was completely abrogated by treatment with U0126. The data suggest that FRS2 was highly threonine phosphorylated in the SUM-52 cells, followed by FGFR2-C3-expressing cells and C1, with no threonine

\section{Affinity precipitate: $\mathrm{p} 13^{\text {suc1 }}$-agarose beads Blot: $\alpha$-phosphotyrosine}
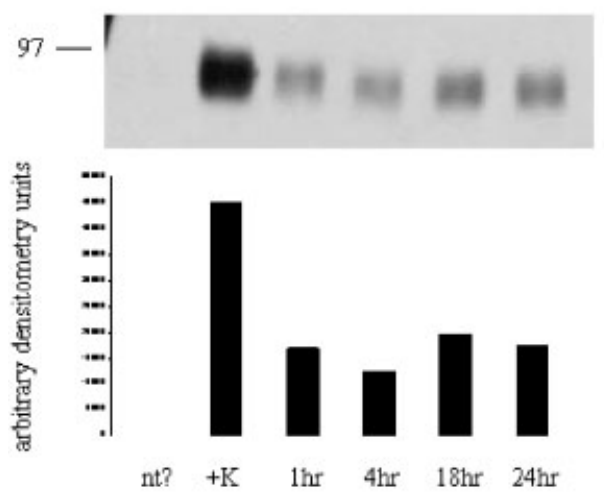

YF\#7

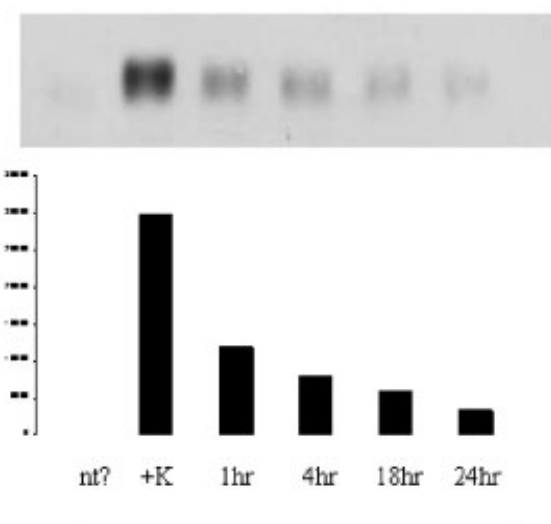

YFpe

Fig. 6. Time course of FRS2 tyrosine phosphorylation after KGF stimulation of FGFR2-C1-Y769F cells. As described in Figure 1, samples were not treated (nt) or treated with KGF and heparin and 10 min (K), then lysed after the indicated number of hours. FRS2 was affinity precipitated and the blots were probed for phosphotyrosine. The graphs below each blot show quantification of band intensities. 


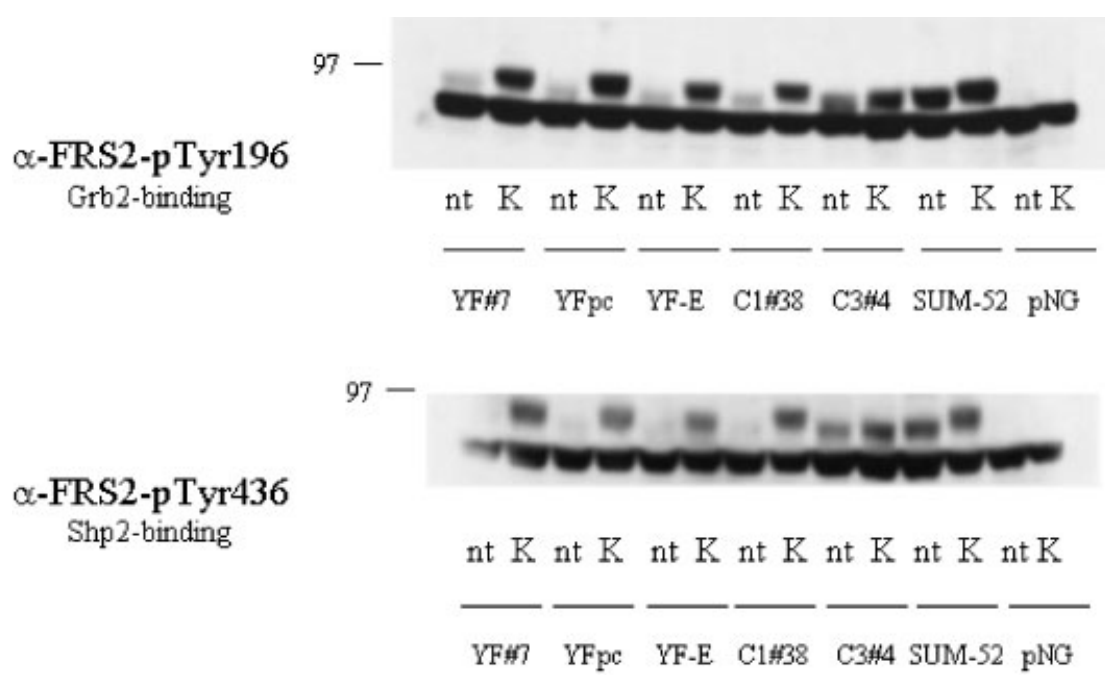

Fig. 7. Phosphospecific FRS2 Western blots in the presence or absence of KGF stimulation. FGFR2-C1Y769F, C1 wild type, C3, pNG, and SUM-52 cells were treated (K) or not (nt) with KGF for 10 min. Whole cell lysate was assessed for levels of pTyr196 (top) or pTyr436 (bottom).

phosphorylation of FRS2 occurring in the pNG empty vector expressing H16N2 cells. Treatment of the FGFR2-C1-expressing cells with KGF induced an increase in the threonine phosphorylation of FRS2, consistent with the increase in FRS2 molecular weight upon ligand stimulation. The data in the phosphotyrosine blot indicate that blocking threonine phosphorylation of FRS2 caused the molecular weight of FRS2 in all cells to be greatly decreased but had no effect on phosphotyrosine levels (Fig. 9, lower). The U0126treated samples show a smear of molecular weight sizes of FRS2, suggesting dephosphorylation occurred on variable numbers of the eight threonine residues. This result suggests that threonine phosphorylation of FRS2 is responsible for a significant molecular weight shift of the protein, as expected. Although different molecular weight sizes are present, the intensity of tyrosine phosphorylation of FRS2 appears to remain unchanged when MAPK was inhibited.

In all, the data presented here strongly suggest that the full-length C3 isoform of FGFR2 expressed by the SUM-52 breast cancer cells is highly transforming to human mammary epithelial cells. Signaling generated by FGFR2-C3 is constitutive, ligand-independent, and robust, as demonstrated by tyrosine phosphorylation levels of the adapter protein FRS2. In contrast, the FGFR2-C1 isoform is dependent on KGF stimulation in order to transiently achieve comparable signaling levels as the C3 isoform. In the presence of KGF, FGFR2-C1 activation of FRS2 is associated with an electrophoretic

\section{Blot: $\alpha-P-E r k$}

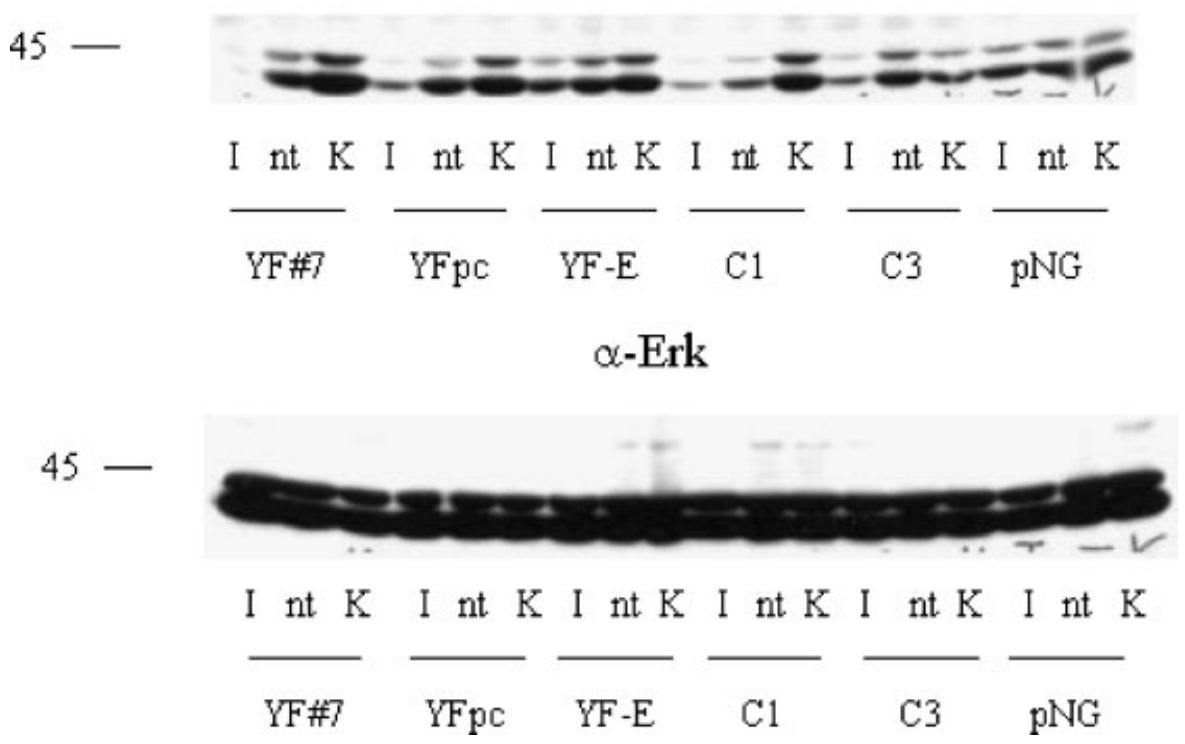

Fig. 8. Erk phosphorylation in cells treated with the FGFR inhibitor PD173074 or stimulated with KGF. FGFR2-C1-Y769F, C1 wild type, C3, and pNG expressing cells were not treated (nt), treated with KGF and heparin for $10 \mathrm{~min}(\mathrm{~K})$, or with the FGFR inhibitor PD173074 for $24 \mathrm{~h}(\mathrm{I})$. Whole cell lysate was assessed for levels of phospho-Erk or Erk-1. 


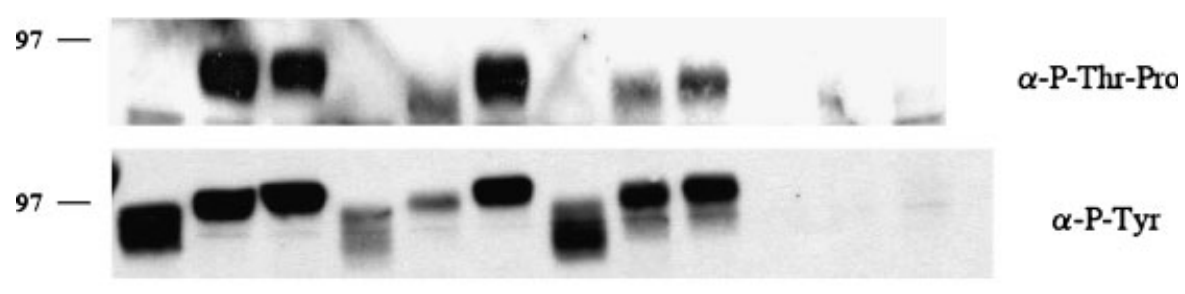

$\frac{\mathrm{U} \text { nt } \mathrm{K}}{\mathrm{SUM}-52} \frac{\mathrm{U} \text { nt } \mathrm{K}}{\mathrm{Cl} \# 38} \frac{\mathrm{C} 3 \# 4}{\mathrm{C} 3 \mathrm{nt}} \frac{}{\mathrm{pNG}}$

Fig. 9. Phosphothreonine analysis of FRS2 in the presence or absence of the MAPK inhibitor U0126. SUM-52, FGFR2-C1, C3, and pNG expressing cells were untreated (nt), treated with $5 \mu$ M of the MAPK inhibitor U0126 for $30 \mathrm{~min}(\mathrm{U})$, or treated with KGF for $10 \mathrm{~min}(\mathrm{~K})$. FRS2 was immunoprecipitated, and the blot was probed for (top) phosphothreonine (pThr-Pro) or phosphotyrosine (lower).

mobility shift not observed following FGFR2-C3 activation of FRS2. Furthermore, mutation of Tyr769 in the C1 carboxyl terminus did not significantly alter the signaling capacity of the FGFR2-C1 isoform and, more importantly, did not result in increased phosphotyrosine levels of FRS2, either constitutively or following ligand stimulation. Finally, in addition to being tyrosine phosphorylated, FRS2 also undergoes threonine phosphorylation in the SUM-52 cells and FGFR2 overexpressing H16N2 cells. Upon MAPK inhibition, threonine phosphorylation of FRS2 was abrogated and the apparent molecular weight was dramatically decreased, without affecting tyrosine phosphorylation levels.

\section{DISCUSSION}

Gene amplification and alternative splicing contribute to the overexpression of nine distinct FGFR2 isoforms in the SUM-52 breast cancer cells. Previous studies of human mammary epithelial cells and NIH3T3 fibroblasts have suggested that overexpression of FGFR2 isoforms with the C3 carboxyl terminus is more transforming than FGFR2-C1 (Itoh et al., 1994; Moffa et al., 2004). In this work, we sought to characterize further the differences in signal transduction by FGFR2-C1 and C3 variants, with specific emphasis on responsiveness to ligand.

The FRS2 adapter protein plays a fundamental role in propagating signals from FGF receptors. Ordinarily, FRS2 gets tyrosine phosphorylated by FGFR upon receptor activation from ligand binding. However, in the SUM-52 breast cancer cells, FRS2 is constitutively highly tyrosine phosphorylated in the absence of ligand. This level of activation can be recapitulated by overexpression of the FGFR2-C3 isoform into H16N2 HME cells. Although FGFR2-C1-expressing cells display phenotypes characteristic of transformation, these cells cannot activate or sustain high levels of FRS2 tyrosine phosphorylation in the absence of KGF, nor do they express the full range of transformed phenotypes. The time course data shown in Figure 1 clearly demonstrate the difference between the basal and KGF-induced tyrosine phosphorylated FRS2 levels in FGFR2-C1 versus C3-expressing cells. Whereas untreated FGFR2-C1-expressing cells do not express detectable levels of tyrosine phosphorylated FRS2, KGF stimulation results in a dramatic increase in FRS2 phosphorylation levels that diminishes with time after KGF removal. The notable distinction in the FGFR2-C3 cells is the robust level of FRS2 tyrosine phosphorylation in non-stimulated cells. Although KGF transiently further increase the level of tyrosine phosphorylated FRS2 in the FGFR2-C3-expressing cells, the signaling potential in the absence of ligand is not susceptible to the same means of downregulation. Furthermore, these data show the electrophoretic mobility shift of FRS2 upon KGF stimulation of FGFR2-C1 cells, whereas the apparent molecular weight of FRS2 remains unchanged in treated versus untreated cells expressing FGFR2-C3.

After accumulating data describing the differences in signaling between FGFR2-C1 and C3, the next logical step was to investigate the structural difference between these isoforms. We focused on tyrosine 769 in the $\mathrm{C} 1$ carboxyl terminus for several reasons. This site is the only known phosphorylated tyrosine present in the C1 carboxyl terminus and absent from C3 (Itoh et al., 1994; Mohammadi et al., 1996). Further, Tyr769 is homologous to Tyr766 on FGFR1, which, upon phosphorylation is known to bind PLC $\gamma$ and/or Shb (Mohammadi et al., 1991; Cross et al., 2002). The absence of this site on FGFR2-C3 suggests that the interactions between FGFR2-C1 and PLC $\gamma$ or Shb could negatively regulate the signaling generated by the FGFR2-C1 isoform. Specifically, the association with Tyr769 and Shb piqued our interest, since Shb has been reported to be involved in activating the MAPK pathway. Evidence in the literature suggests that threonine phosphorylation of FRS2 by MAPK decreases FRS2 tyrosine phosphorylation (Lax et al., 2002). Together, this led to our hypothesis that activation of MAPK by FGFR2-C1 via Shb stimulates threonine phosphorylation of FRS2, leading to the rapid loss of tyrosine phosphorylated FRS2 observed following KGF stimulation. Therefore, we hypothesized that mutating Tyr769 to a phenylalanine would lead to a version of FGFR2-C1 with a reduced ability to activate MAPK resulting in decreased levels of threonine phosphorylated FRS2, and enhanced levels of tyrosine phosphorylated FRS2, making it more similar to FGFR2-C3.

Our data analyzing the $\mathrm{C} 1-\mathrm{Y} 769 \mathrm{~F}$ mutant indicate that disrupting the predicted PLC $\gamma$ and Shb interaction site did not enhance FGFR2-C1 signaling activity. Rather, the C1-Y769F mutant was as transforming to HME cells as the wild type FGFR2-C1, and was able to similarly activate FRS2. Neither the wild type nor mutant FGFR2-C1 isoform stimulated basal FRS2 
tyrosine phosphorylation to levels as robust as observed in FGFR2-C3-expressing cells. This suggests that Tyr769 alone does not affect the ability of FGFR2-C1 to induce and maintain downstream signaling pathway activation. Even when overexpressed, the wild type or mutant FGFR2-C1 isoform behaved in a manner more similar to normal, controlled receptor signaling, as opposed to the constitutive, ligand-independent signaling activated by the FGFR2-C3 isoform.

Prior studies have described mutating the homologous PLC $\gamma$-binding site on FGFR1, Tyr766, and suggested that FGFR1 signaling does not depend on activation of this pathway. Mohammadi et al. (1992) and Peters et al. (1992) originally showed that mutation of Tyr766 on FGFR1 led to the expected decrease in PLC $\gamma$ activity and decreased activation of protein kinase C, without abrogating FGFR1-mediated mitogenesis. In addition, FGFR1 site-mutated at Tyr766 remained capable of inducing chemotaxis of endothelial cells and neuronal differentiation of PC12 cells, as reported by Clyman et al. (1994), Spivak-Kroizman et al. (1994), and Landgren et al. (1998). Concurrent with our generating and studying FGFR2-C1-Y769F, Ceridono et al. (2005) reported generation of the same mutant, which they overexpressed in NIH3T3 and HeLa cells. Their data suggested that the FGFR2-Y769F mutation negatively affected phosphorylation of FRS2 by FGFR2-C1 and activation of MAPK, yet did not change the endocytosis of FGFR2. In contrast, Sorokin et al. (1994) published data indicating that Tyr766 on FGFR1 was involved in the induction of receptor internalization, downregulation, and degradation.

As mentioned above, our data show that FGFR2-C1 and C3-expressing cells exhibit a considerable difference in their levels of tyrosine phosphorylated FRS2. To further analyze the activation of the FRS2 adapter protein, we also examined threonine phosphorylation. We found that the levels of FRS2 threonine phosphorylation were lower in the FGFR2-C1-expressing cells, as compared to FGFR2-C3 and SUM-52 cells. In the presence of KGF stimulation, molecular weight and threonine phosphorylation levels of FRS2 were dramatically increased in the FGFR2-C1-expressing cells, and not FGFR2-C3. The threonine phosphorylation of FRS2 in all of the cells is dependent upon MAPK activity. The comparably low level of basal FRS2 threonine phosphorylation in the FGFR2-C1 cells was unexpected in light of the data in the literature. Lax et al. (2002) proposed that FRS2 threonine phosphorylation is mutually exclusive with tyrosine phosphorylation of the protein, thus hypothesizing that threonine phosphorylation of FRS2 decreases its ability to serve as a docking molecule for downstream signal activation. Rather than a mutual exclusivity between threonine and tyrosine phosphorylation of FRS2 in our cells, we observed proportionality. The cells with the highest level of tyrosine phosphorylated FRS2, SUM-52, also expressed the highest level of threonine phosphorylated FRS2. Further, tyrosine phosphorylation levels of FRS2 remained the same regardless of changes to threonine phosphorylation status that resulted from MAPK inhibition (Fig. 9).

These important observations led to a hypothesis proposing that FGFR2-C1 and FGFR2-C3 modify the FRS2 adapter protein in a fundamentally different manner. There are six tyrosine and eight threonine residues on FRS2 that are known to be sites for phosphorylation (Kouhara et al., 1997; Hadari et al., 1998; Lax et al., 2002). We propose that FGFR2-C1 and FGFR2-C3 activate these sites in an inherently different way, leading to differential downregulation of tyrosine phosphorylated FRS2, possibly via ubiquitin-mediated degradation or dephosphorylation. This is consistent with the observation that the level of tyrosine phosphorylated FRS2 persists at a high intensity in the FGFR2-C3-expressing cells. We observed KGF-induced increases in FRS2 tyrosine phosphorylation in both FGFR2-C1- and C3-expressing cells, with levels decreasing after ligand removal. However, the high basal level of tyrosine phosphorylated FRS2 in the FGFR2-C3 cells remained unchanged, suggesting that a different mechanism of FRS2 activation and deactivation is employed by cells expressing the FGFR2-C3 isoform whether in the presence or absence of ligand. By contrast, the FGFR2-C1 isoform is only fully activated in a ligand-dependent manner. Additionally, tyrosine phosphorylated FRS2 in KGF-stimulated FGFR2-C1 cells migrates at a higher apparent molecular weight than FRS2 in the FGFR2-C3 cells, even in the presence of KGF. This would further suggest that phosphorylation, or other posttranslational events, might be taking place on FRS2 in the FGFR2-C1 cells differently from the C3-expressing cells, resulting in rapid downregulation of tyrosine phosphorylated FRS2. Intensive proteomic analyses of FRS2 under different conditions in the FGFR2-C1- and C3-expressing cells could reveal the different modifications of FRS2 by each receptor isoform.

Normal human mammary epithelial cells express only full-length FGFR2 isoforms with the C1 carboxyl terminus. In contrast, the SUM-52 human breast cancer cells highly overexpress nine distinct isoforms of FGFR2, which include three different carboxyl termini. Overexpression of the full-length FGFR2-C3 isoform into HME cells leads to robust activation of downstream signaling, as evidenced by high levels of constitutively tyrosine phosphorylated FRS2 in the absence of ligand, whereas cells expressing FGFR2-C1 required constant stimulation with $\mathrm{KGF}$ to achieve and maintain similar activation. It is important to recall that the $\mathrm{C} 1$ and $\mathrm{C} 3$ carboxyl termini are expressed by different exons; C3 is not a truncated version of C1 (Itoh et al., 1994). Clearly, this FGFR2 alternative splicing event allowing the inclusion of the exon for the C3 carboxyl terminus conferred a growth advantage contributing to the transformation of the SUM-52 breast cancer cells. The FGFR2-C1 and C3 variants studied here are identical in their ligand-binding domain, yet display dramatically different dependency upon ligand for activating downstream signal transduction. Therefore, these data strongly suggest that the specific presence of FGFR2 isoforms with the C3 carboxyl terminus is fundamental to the transformed phenotypes of the SUM-52 cells, and other cancer cells in which alternatively spliced FGFR2 is activated by gene amplification.

\section{LITERATURE CITED}

Adnane JGP, Dionne CA, Crumley G, Jaye M, Schlessinger J, Jeanteur P, Birnbaum D, Theillet C. 1990. BEK and FLG, two receptors to members of the FGF family, are amplified in subsets of human breast cancers. Oncogene 6:659-663.

Band V, Zajchowski D, Swisshelm K, Trask D, Kulesa V, Cohen C, Connolly J, Sager R. 1990. Tumor progression in four mammary epithelial cell lines derived from the same patient. Cancer Res 50:7351-7357.

Cappellen D, De Oliveira C, Ricol D, de Medina S, Bourdin J, Sastre-Garau X Chopin D, Thiery JP, Radvanyi F. 1999. Frequent activating mutations of FGFR3 in human bladder and cervix carcinomas. Nat Genet 23:18-20.

Ceridono M, Belleudi F, Ceccarelli S, Torrisi MR. 2005. Tyrosine 769 of the keratinocyte growth factor receptor is required for receptor signaling but not endocytosis. Biochem Biophys Res Commun 327:523-532.

Clyman RI, Peters KG, Chen YQ, Escobedo J, Williams 1994. Phospholipase $\mathrm{C}$ gamma activation, phosphotidylinositol hydrolysis, and calcium mobilization are not required for FGF receptor-mediated chemotaxis. Cell Adhes Commun 1:333-342. 
Cross MJ, Lu L, Magnusson P, Nyqvist D, Holmqvist K, Welsh M, ClaessonWelsh L. 2002. The Shb adaptor protein binds to tyrosine 766 in the FGFR-1 and regulates the Ras/MEK/MAPK pathway via FRS2 phosphorylation in endothelial cells. Mol Biol Cell 13:2881-2893.

Ethier SP, Kokeny KE, Ridings JW, Dilts CA. 1996. erbB family receptor expression and growth regulation in a newly isolated human breast cancer cell line. Cancer Res 56:899-907.

Hadari YR, Kouhara H, Lax I, Schlessinger J. 1998. Binding of Shp2 tyrosine phosphatase to FRS2 is essential for fibroblast growth factor-induced PC12 cell differentiation. Mol Cell Biol 18:3966-3973.

Hattori Y, Odagiri H, Nakatani H, Miyagawa K, Naito K, Sakamoto H, Katoh O, Yoshida T, Sugimura T, Terada M. 1990. K-sam, an amplified gene in stomach cancer, is a member of the heparin-binding growth factor receptor genes. Proc Natl Acad Sci USA 87:5983-5987.

Itoh H, Hattori Y, Sakamoto H, Ishii H, Kishi T, Sasaki H, Yoshida T, Koono M, Sugimura T, Terada M. 1994. Preferential alternative splicing in cancer generates a K-sam messenger RNA with higher transforming activity. Cancer Res 54:3237-3241.

Jang J-H SK-H, Park J-G. 2001. Mutations in fibroblast growth factor receptor 2 and fibroblast growth factor receptor 3 genes associated with human gastric and colorectal cancers. Cancer Res 61:3541-3543.

Kouhara H, Hadari YR, Spivak-Kroizman T, Schilling J, Bar-Sagi D, Lax I, Schlessinger J. 1997. A lipid-anchored Grb2-binding protein that links FGF-receptor activation to the Ras/MAPK signaling pathway. Cell 89:693702.

Landgren E, Klint P, Yokote K, Claesson-Welsh L. 1998. Fibroblast growth factor receptor-1 mediates chemotaxis independently of direct SH2-domain protein binding. Oncogene 17:283-291.

Lax I, Wong A, Lamothe B, Lee A, Frost A, Hawes J, Schlessinger J. 2002. The docking protein FRS2alpha controls a MAP kinase-mediated negative feedback mechanism for signaling by FGF receptors. Mol Cell 10:709-719.

Moffa AB, Tannheimer SL, Ethier SP. 2004. Transforming potential of alternatively spliced variants of fibroblast growth factor receptor 2 in human mammary epithelial cells. Mol Cancer Res 2:643-652.
Mohammadi M, Honegger AM, Rotin D, Fischer R, Bellot F, Li W, Dionne CA Jaye M, Rubinstein M, Schlessinger J. 1991. A tyrosine-phosphorylated carboxy-terminal peptide of the fibroblast growth factor receptor (Flg) is a binding site for the SH2 domain of phospholipase C-gamma 1. Mol Cell Biol 11:5068-5078.

Mohammadi M, Dionne CA, Li W, Li N, Spivak T, Honegger AM, Jaye M Schlessinger J. 1992. Point mutation in FGF receptor eliminates phosphatidylinositol hydrolysis without affecting mitogenesis. Nature 358:681-684.

Mohammadi M DI, Sorokin A, Burgess WH, Jaye M, Schlessinger J. 1996. Identification of six novel autophosphorylation sites on fibroblast growth factor receptor 1 and elucidation of their importance in receptor activation and signal transduction. Mol Cell Biol 16:977-989.

Penault-Llorca F BF, Adelaide J, Parc P, Coulier F, Jacquemier J, Birnbaum D DeLapeyriere O. 1995. Expression of FGFand FGFreceptor genes in human breast cancer. Int J Cancer 61:170-176.

Peters KG, Marie J, Wilson E, Ives HE, Escobedo J, Del Rosario M, Mirda D, Williams LT. 1992. Point mutation of an FGF receptor abolishes phosphatidylinositol turnover and $\mathrm{Ca} 2+$ flux but not mitogenesis. Nature 358:678-681.

Shin EY, Lee BH, Yang JH, Shin KS, Lee GK, Yun HY, Song YJ, Park SC, Kim EG. 2000. Up-regulation and co-expression of fibroblast growth factor receptors in human gastric cancer. J Cancer Res Clin Oncol 126:519-528.

Sorokin A, Mohammadi M, Huang J, Schlessinger J. 1994. Internalization of fibroblast growth factor receptor is inhibited by a point mutation at tyrosine 766. J Biol Chem 269:17056-17061.

Spivak-Kroizman T, Mohammadi M, Hu P, Jaye M, Schlessinger J, Lax I. 1994 Point mutation in the fibroblast growth factor receptor eliminates phosphatidylinositol hydrolysis without affecting neuronal differentiation of PC12 cells. J Biol Chem 269:14419-14423.

Tannheimer SL RA, Ethier SP. 2000. Characterization of fibroblast growth factor receptor 2 overexpression in the human breast cancer cell line SUM-52PE. Breast Cancer Res 2:311-320.

$\mathrm{Xu}$ H, Lee KW, Goldfarb M. 1998. Novel recognition motif on fibroblast growth factor receptor mediates direct association and activation of SNT adapter proteins. J Biol Chem 273:17987-17990. 